\title{
CD80-CD28 signaling controls the progression of inflammatory colorectal carcinogenesis
}

\author{
Marco Scarpa ${ }^{1, *}$, Paola Brun ${ }^{2, *}$, Melania Scarpa ${ }^{1}$, Susan Morgan ${ }^{3}$, Andrea Porzionato ${ }^{2}$, \\ Andromachi Kotsafti ${ }^{1}$, Marina Bortolami ${ }^{4}$, Andrea Buda ${ }^{4}$, Renata D'Incà ${ }^{4}$, Veronica \\ Macchi $^{2}$, Giacomo C. Sturniolo ${ }^{4}$, Massimo Rugge ${ }^{5}$, Romeo Bardini ${ }^{4}$, Ignazio \\ Castagliuolo², Imerio Angriman $^{4, \S}$, Carlo Castoro ${ }^{1, \S}$ \\ ${ }^{1}$ Oncological Surgery Unit, Veneto Institute of Oncology IOV - IRCCS, Padova 35128, Italy \\ ${ }^{2}$ Department of Molecular Medicine, University of Padova, Padova 35128 Italy \\ ${ }^{3}$ Department of Histopathology, Sheffield Teaching Hospitals, Sheffield S10 2JF, UK \\ ${ }^{4}$ Department of Surgery Oncology and Gastroenterology, University of Padova, Padova 35128, Italy \\ ${ }^{5}$ Department of Medicine, University of Padova, Padova 35128, Italy \\ *These authors have contributed equally to this work \\ $\S$ These senior authors contributed equally to this work \\ Correspondence to: \\ Marco Scarpa, e-mail: marcoscarpa73@yahoo.it \\ Keywords: ulcerative colitis, colorectal carcinogenesis, antigen presenting cells, CD8+ T cells, intestinal epithelial cells \\ Received: September 18, $2014 \quad$ Accepted: November 19, $2014 \quad$ Published: January 16, 2015
}

\section{ABSTRACT}

In patients with ulcerative colitis (UC) the cumulative risk of colon cancer is lower than the actual rate of dysplasia suggesting an efficient immune surveillance mechanism. Since the co-stimulatory molecule CD80 is overexpressed in dysplastic colonic mucosa of UC patients and T-cell activation entails effective costimulation, we aimed to evaluate the functional implication of CD80 signaling in colonic UC-associated carcinogenesis. In humans, we observed that the percentage of CD80+ and HLA-A+ IEC was increased in the dysplastic colonic mucosa of UC patients. In vitro, IEC activated CD8+ T-cells through a CD80-dependent pathway. Finally, in the AOM/DSS-induced colonic adenocarcinoma model CD80 signaling inhibition significantly increased the frequency and extension of high-grade dysplasia, whereas enhancing CD80 activity with an anti-CTLA4 antibody significantly decreased colonic dysplasia. In conclusion, CD80 signaling between IEC and T-cells represents a key factor controlling the progression from low to high grade dysplasia in inflammatory colonic carcinogenesis.

\section{INTRODUCTION}

Ulcerative colitis (UC) is a chronic inflammatory disorder involving the rectum and to a various extent the colon [1]. UC patients experience several complications including an increased risk of colorectal cancer (CRC) [1]. Several independent risk factors for malignancy in UC patients have been identified such as duration of disease, extent of inflammation, history of concurrent primary sclerosing cholangitis and family history of CRC [2]. Pre-malignant histological alterations in UC patients are broadly referred to as dysplasia, rather than adenoma, since dysplasia is frequently not polypoid [3, 4]. Moreover, several molecular pathways such as wnt- $\beta$ catenin pathway are involved in colonic carcinogenesis in UC [5]. Current guidelines for management of dysplasia in $\mathrm{UC}$ recommend total proctocolectomy in case of high grade dysplasia (HGD) or in case of two consecutive observation of low grade dysplasia (LGD) [6]. Intriguingly, the cumulative risk of colon cancer in patients with UC is approximately $8 \%$ twenty years after the diagnosis, although the cumulative rate of dysplasia is at least $25 \%$ [7-8]. Moreover, proctocolectomy specimens from UC patients with preoperative diagnosis of dysplasia exhibit neoplastic lesions in only two third of cases [9], and $64 \%$ of UC patients with LGD exhibit indefinite or no dysplasia after 4-years follow-up [10]. These inconsistencies show a fluid and variable presence of dysplastic lesions in the inflamed colonic mucosa indicating the possibility of a regression of preneoplastic lesions. These observations suggest the presence of efficient 
immune surveillance mechanisms that lead to neoplastic cells destruction (immune surveillance), possibly mediated by T-cells, macrophages, and natural killer cells [11].

Successful T-cell activation entails effective costimulation signaling mediated by CD80, CD86 or CD40 on the antigen-presenting cells (APC) and CD28 or CD40L receptors on T-cells [12-14]. CTLA4, a member of the immunoglobulin superfamily expressed on the T-cell surface, can compete with CD28 for binding to CD80 and CD86, transmitting an inhibitory signal to $\mathrm{T}$ cells instead [15]. CD80 expression can be induced by oncogenic insults [16], including oxidative DNA damage associated to intestinal chronic inflammation [17], suggesting a major role in immune surveillance mechanisms. In a previous study, we reported CD80 mRNA upregulation in UC preneoplastic lesions and its down-regulation in invasive cancer $[17,18]$. However, the precise role of CD80 signaling and its regulation during UC-related colonic carcinogenesis remains unclear.

Several studies have investigated T-cell activation in $\mathrm{CRC}$ but few data are available concerning its role in the early stages of inflammatory carcinogenesis. A significantly higher proportion of activated CD8 T-cells expressing CD69 and CD107 in early invasive cancer compared to advanced cancer in non inflammatory conditions has been described [19]. Pages et al., meanwhile, showed that non inflammatory $\mathrm{CRC}$ without signs of early metastatic invasion have increased infiltrates of immune cells and higher levels of downstream products of Th1 activation but not of inflammatory or immunosuppressive mediators [20]. However, a clear characterization of the immune microenvironment, and in particular of $\mathrm{T}$ cells activation, in colonic inflammatory carcinogenesis is still lacking. Therefore, the aim of our study was to evaluate the functional implication of CD80 signaling in the epithelialCD8+T-cells cross-talk in immune surveillance mechanisms during UC-associated carcinogenesis of the colon.

\section{RESULTS}

\section{Human epithelial cells can act as antigen presenting cells in UC-related colonic carcinogenesis}

In our series, CD80 mRNA peaked in mucosal samples from patients with UC and dysplasia and resulted down regulated in patients with UC and cancer (Supplementary Figure 1). In normal human colonic mucosa only a small proportion of cytokeratin-20-positive epithelial cells $(\mathrm{CK} 20+)$ expresses the co-stimulatory molecule CD80, or HLA-ABC or HLA-DR (Figure 1A and $1 \mathrm{~B})$. However, in UC-related carcinogenesis, the percentage of CK20+ expressing either CD80+, HLA$\mathrm{ABC}+$ or HLA-DR + increased and peaked in the dysplastic colonic mucosa (Figure 1A and 1B). On the other hand, CD40 expression remained substantially unvaried along the carcinogenic pathway (Figure 1B). Furthermore, the rate of $\mathrm{CD} 80+$ epithelial cells was significantly higher in UC patients with dysplasia compared with non UC-related dysplasia (Figure 1A).

\section{T-cell activation in human colonic carcinogenesis}

To determine the level of $\mathrm{T}$ cells activation in human colonic carcinogenesis, qRT-PCR and immunohistochemistry
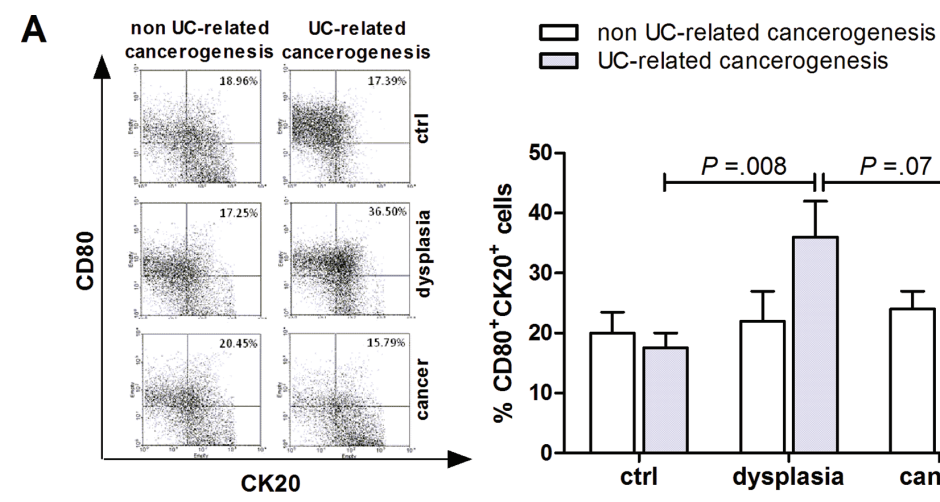

B

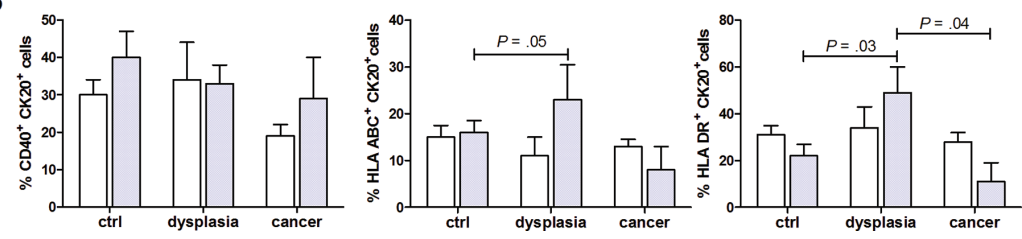

Figure 1: Characterization of CD80 expression on epithelial cells in human colon carcinogenesis. Flow cytometric analysis of human colonic mucosa specimen showing the proportion of epithelial (Cytokeratin 20, Ck20+) cells expressing CD80 (A) and CD40, HLA-ABC or HLA-DR (B) in non UC-related and UC-related carcinogenesis. Data are presented as the mean \pm S.E.M. Kruskal-Wallis test was used for comparisons. 
were used to quantitate CD69 (pan T cells activation marker) and CD107 (marker of actively degranulating cells). CD69 mRNA was significantly higher in patients with UC and dysplasia than in UC or UC and cancer (Figure 2A). Moreover, infiltration of CD107+ cells was higher in UC patients with dysplasia than in UC patients or UC with cancer (Figure 2B).

Next, we characterized the cytotoxic $T$ lymphocytes subgroup along the steps of human colon carcinogenesis. CD8+ T-cells were activated in presence of dysplastic colonic mucosa in UC patients, as demonstrated by the enhanced proportion of CD8+ lymphocytes expressing the early activation marker CD38 (Figure 3B), the high affinity CD8 receptor CD $8 \beta$ and the cytotoxin TNF $\beta$ (Figure 3D and 3E). Intriguingly, CD8+ lymphocytes expressing CD28, the CD80 receptor, were reduced in UC patients who developed colon cancer as opposed to patients with UC and dysplasia (Figure 3A). The synchronous presence of CD80+ epithelial cells and activated CD8+ T-cells (rho $=0.65, p=0.001$ ) suggests that the immune surveillance mechanism in UC may be mediated by the direct interaction between epithelial cells and infiltrating CD8+ T-cells (Figure 3C).

By contrast with the CD8+ lymphocytes activation described above, CD4+ lymphocytes infiltrating the colonic mucosa did not show sign of activation along the steps of UC-related carcinogenesis (Supplementary Figure 3). Indeed, T-bet, a T-box transcription factor expressed in CD4+ $\mathrm{T}$ lymphocytes committed to Th1 phenotype, and IL-12, a stimulator of naive $\mathrm{T}$ cells differentiation into Th1 cells, did not show any significant alteration along UC-related carcinogenesis pathways (Supplementary Figure 3A and 3C). Both these markers resulted higher in the adenoma than in the non UC-related cancer. IL-17, a marker of activity of Th17 subpopulation, resulted unvaried along the steps of UC-related carcinogenesis while its expression tended to be higher in the adenoma than in the healthy mucosa (Supplementary Figure 3B). Finally, IL-35, a Treg marker of activity, did not show any variation along UC-related carcinogenesis (Supplementary Figure 3D).

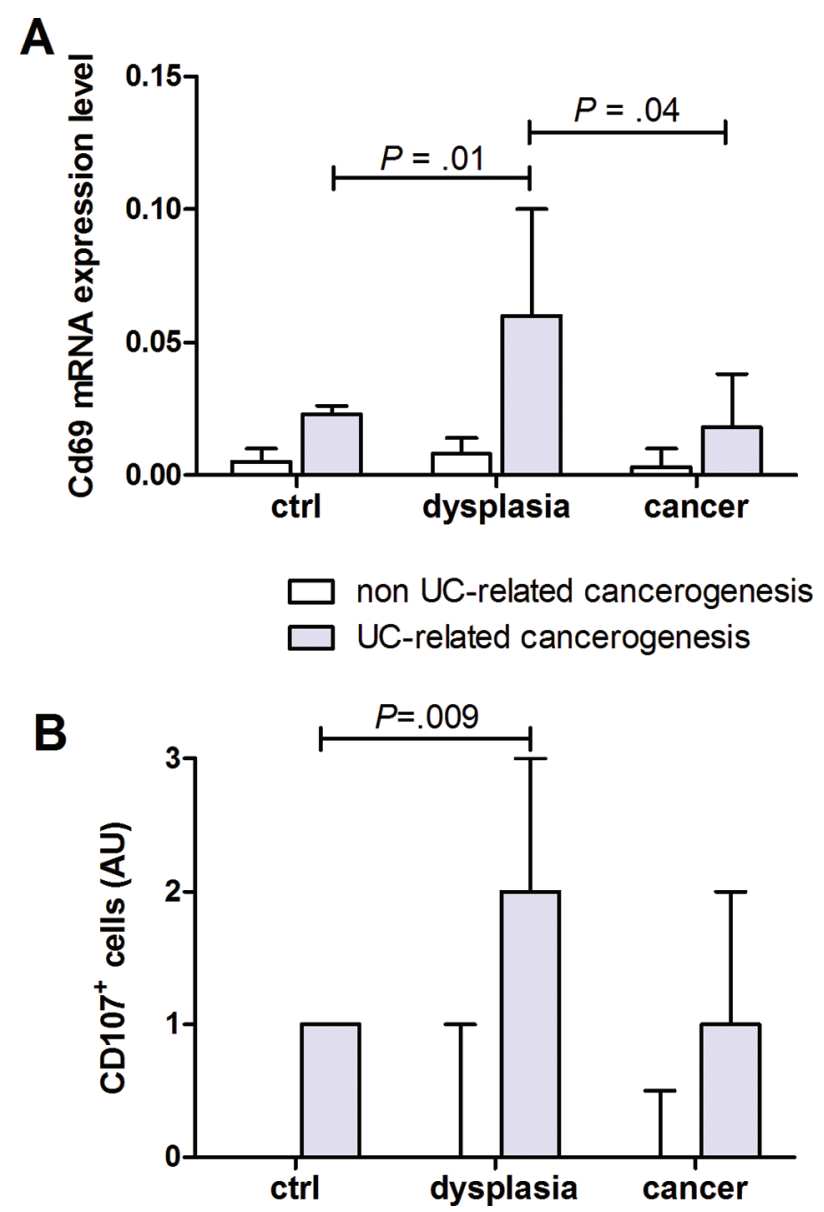

Figure 2: T cells activation in human colon carcinogenesis. Quantification of (A) CD69 mRNA and (B) immunohistochemical quantification of CD107+ cells in human colonic mucosa specimen at different stages of non UC-related and UC-related carcinogenesis. Data are presented as the mean \pm S.E.M. Kruskal-Wallis test was used for comparisons. 


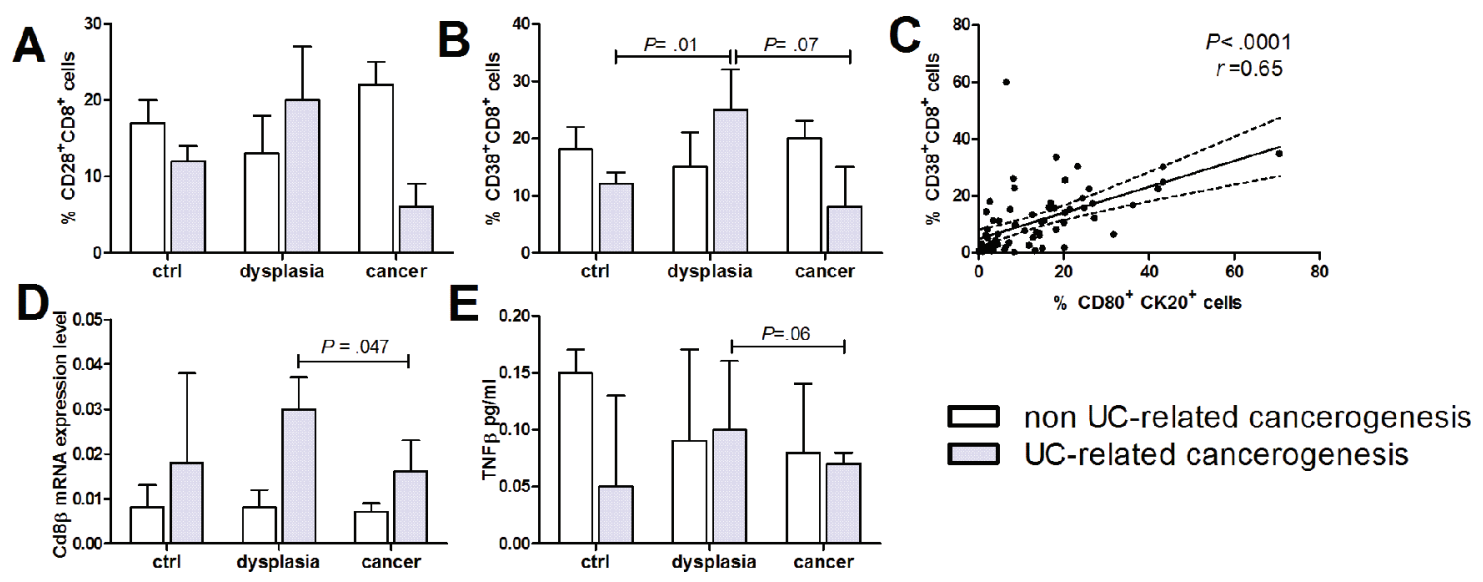

Figure 3: CD8+ lymphocytes infiltration and activation in human colon carcinogenesis. Human intestinal mucosa specimen of non UC-related and UC-related carcinogenesis were tested for flow cytometric quantification of (A) CD28+ and (B) CD38+ CD8+ cells. (C) Correlation between CD80+ human intestinal epithelial cells and activated CD8 lymphocytes. (D) CD8 $\beta$ mRNA levels by RT-qPCR, (E) TNF $\beta$ protein levels. Data are presented as the mean \pm S.E.M. Kruskal-Wallis test was used for comparisons.

\section{In vitro CD8 T-cell activation is mediated by CD80-CD28 signaling}

The simultaneous presence of activated CD8+ T-cells and CD80+ epithelial cells suggested that a direct interaction between these cells might be required for an effective immune surveillance process. Thus, we evaluated whether intestinal epithelial cells isolated from healthy colonic mucosa (obtained far from active inflammation or neoplasm) could induce a CD80-dependent activation of syngeneic lymphocytes extracted from pericolonic lymph nodes. Mucosa of patients undergoing elective operations for colonic diverticular disease or Crohn's disease (non neoplastic group) and for colonic adenocarcinoma (neoplastic group) was used. As shown in Figure 4A, epithelial cells isolated from colon of patients with inflammatory disorders provoked a $40 \%$ increase in the proportion of activated CD8+ T-cells (CD38+), that was completely blocked by anti-CD80 antibody. By contrast, in case of epithelial cells derived from patients with a colonic adenocarcinoma, there was no significant $\mathrm{CD} 8+$ activation (Figure 4A). Furthermore, epithelial cells co-culture triggered $\mathrm{CD} 4+\mathrm{T}$ cells activation independently from CD80 signaling (Figure 4B).

\section{CD80 expression peaks in dysplastic epithelium in a mouse model of colonic carcinogenesis}

Next, we characterized CD80 expression in the AOM/DSS colonic inflammatory carcinogenesis mouse model (Figure 5A). Like in UC patients, there was a patchy distribution of CD80 on epithelial cells and mononucleated cells within lamina propria (Figure 5F). However, whereas CD80+ lamina propria mononucleated cells were homogeneously distributed in the presence of normal or dysplastic epithelium, CD80+ epithelial foci were significantly more frequent in the colonic crypts with LGD or HGD and in the colonic luminal surface with HGD compared with the normal epithelium (Figure 5F). In addition, we determined the time course expression of CD80 in our model. As shown in Figure 5B, the percentage of $\mathrm{CD} 80+$ epithelial cells showed a peak on day 0 , then it exhibited a nadir at day 7 , followed by another peak of expression at day 28. The inflammatory score showed a similar trend over time with a significant peak at day 28 (Figure 5C). On the contrary, HGD extension peaked at day 7 and decreased at day 28 and 56 (Figure 5E) while LGD extension was almost constant along all the steps of the experiment (Figure 5D). Notably, by day 28 approximately half of the mice spontaneously cleared HGD (Figure 5E), evidencing that in this model physiological immune surveillance is occurring.

\section{CD80-CD28 signaling controls murine colonic inflammatory carcinogenesis}

To demonstrate the functional role of CD80 in immune surveillance during carcinogenesis progression, at the end of the AOM-DSS colitis protocol we administered neutralizing antibodies against either CD80 or CTLA4 to inhibit or enhance CD80 signaling, respectively [19] and against $\mathrm{CD} 8$ to assess the role of cytotoxic $\mathrm{T}$ cells in this process (Figure 6A-6B). In line with the role of $\mathrm{CD} 80+$ cells in colitis [26, 27], the inflammatory score was significantly lower in mice treated with anti-CD80 but was not affected in mice treated with anti-CTLA4 (Figure 6C). Consistent with a pivotal role for CD80 in cancer immune surveillance, blocking CD80 signaling caused a significant increase 
A

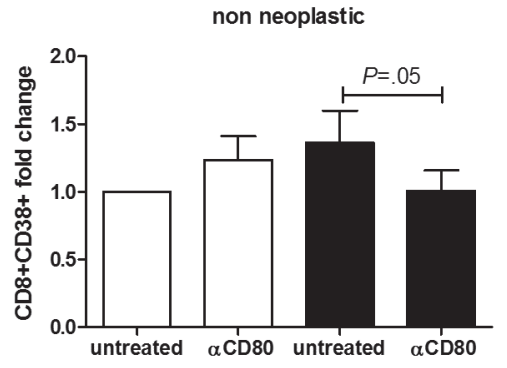

B

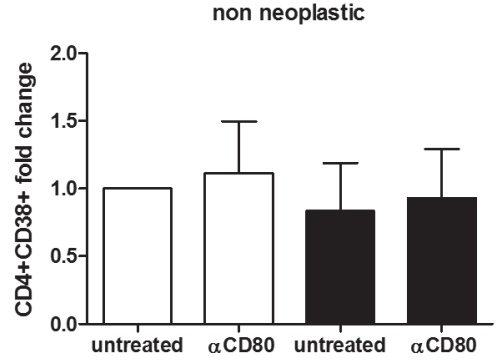

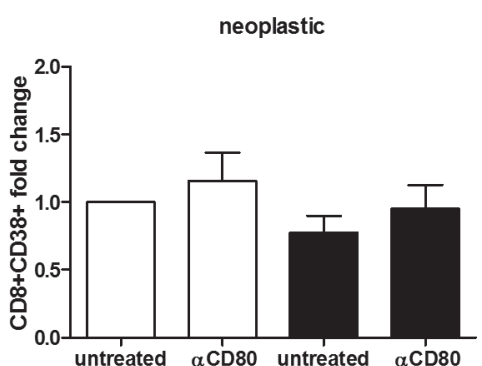

MLN

$M L N+I E C$

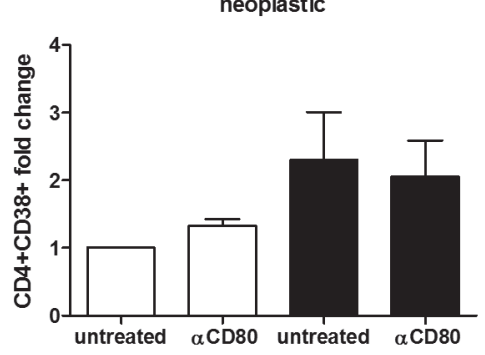

Figure 4: Effect of in vitro CD80 neutralization on lymphocytes activation. MLN lymphocytes were cocultured 24 hrs with human intestinal epithelial cells derived from healthy colonic mucosa of non neoplastic and neoplastic patients $(n=5-12)$. Flow cytometric quantification of $\mathrm{CD} 38+\mathrm{CD} 8+(\mathbf{A})$ and $\mathrm{CD} 38+\mathrm{CD} 4+(\mathbf{B})$ cells is shown. Data are presented as the mean \pm S.E.M. The Kruskal-Wallis ANOVA followed by Wilcoxon paired test were used for comparisons.

in HGD frequency and extension $(p=0.01$ and $p=0.04$, respectively), whereas the number and total area of HGD foci were minimal in anti-CTLA4-treated mice (Figure 6D). Moreover, blocking CD8 signaling caused an increase in both LGD and HGD extension and frequency, mimicking that caused by CD80 inhibition (Figure 6D).

\section{CD80-CD28 signaling shapes immune responses in experimental inflammation driven colon carcinogenesis}

Because the main immune surveillance mechanisms seemed to occur at an early stage following the inflammatory insult in the AOM-DSS model of colonic inflammatory-mediated carcinogenesis, we analyzed the immunological events taking place at this time point (day 7). Anti-CD80 administration caused a significant increase in $\mathrm{CD} 28+\mathrm{CD} 8+$ lymphocyte (Figure 7A). Moreover, anti-CTLA4 treatment increased the proportion of $\mathrm{CD} 80+$ epithelial cell and the expression of the activation marker CD69 on CD8+ $\mathrm{T}$ cells (Figure 7A). In fact, the massive presence of CD8+ lymphocytes in dysplastic areas suggests that these cells may be the main executors of the CD80 immune surveillance-driven process in the colon. Indeed, the number of early-activated lymphocytes (CD38+ and $\mathrm{CD} 69+)$ is reduced in dysplastic lesions of mice treated with anti-CD80 as opposed to the anti-CTLA4-treated mice (Figure 7B and 7C).

\section{DISCUSSION}

Formulated in the early seventies [21], the immune surveillance theory has always been difficult to demonstrate. Dunn and co-workers [22] revamped it by proposing the cancer immunoediting theory, according to which a tumour interacts with the immune system in three sequential steps: an elimination phase during which the immune system clears out most tumour cells; an equilibrium phase during which an adaptive immune response maintains occult cancer in an equilibrium state; and finally, an escape phase during which a tumour grows, invades, and metastasizes [23]. Pages and co-workers demonstrated that in non-inflammatory $\mathrm{CRC}$ an increase in T-cell migration, activation and differentiation ensures a sort of equilibrium phase in tumours without vascular embolism, lymphatic invasion or perineural infiltration [24]. In UC, the discrepancy between the dysplasia rate and the actual cancer incidence suggests that an elimination phase may actively prevent progression from dysplasia to cancer. We postulate that $\mathrm{CD} 80$ over-expression, linked to oxidative DNA damage in the colonic mucosa of UC patients with dysplasia, may play an important role during this elimination phase [18, 19, 25].

Our results suggest that colonic epithelial cells may act as non professional APCs in UC-related colonic carcinogenesis through CD80, HLA-ABC and HLA-DR upregulation. Interestingly, a recent study showed that at least 20 genes, including cancer-related ones, had a 

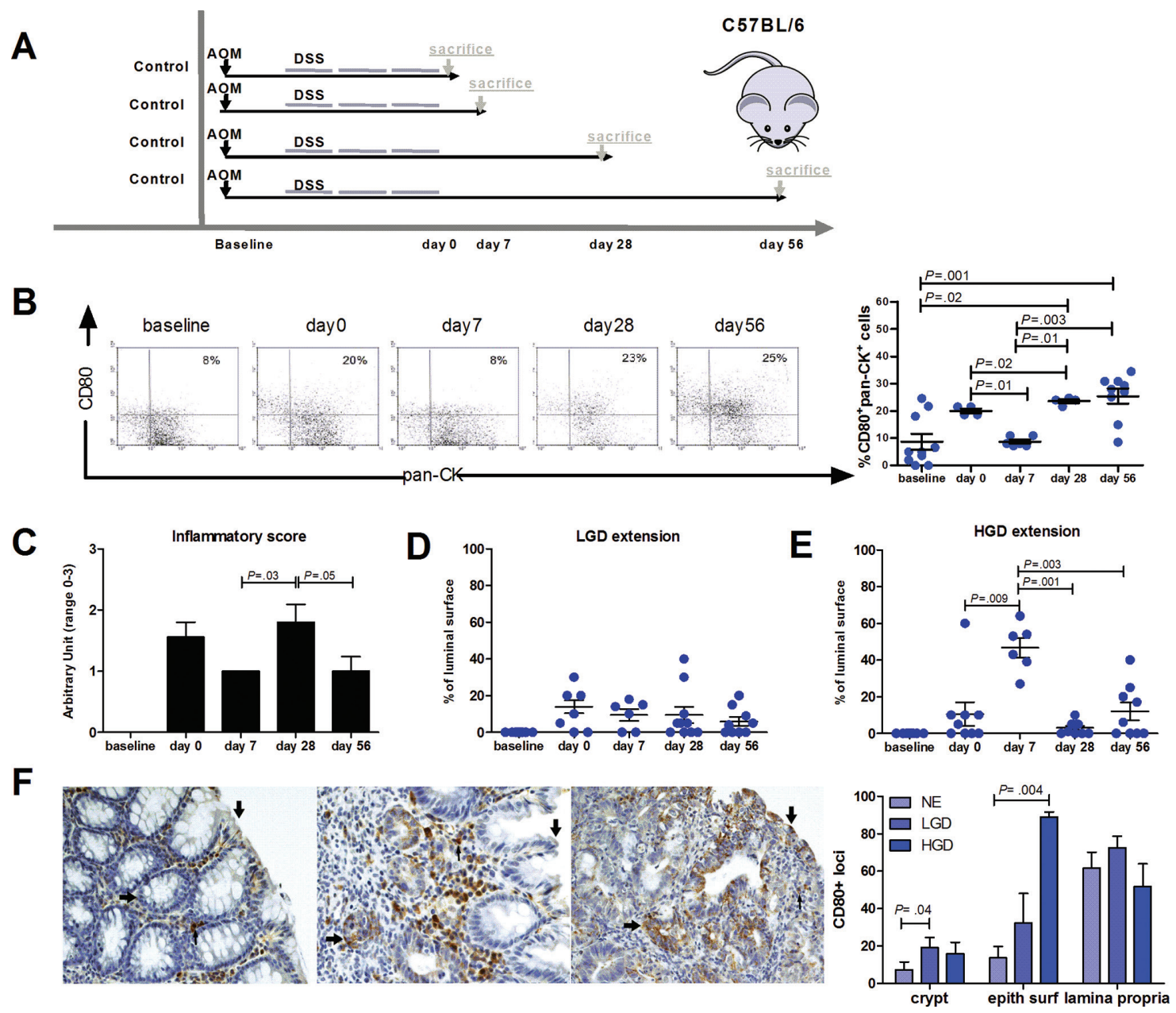

Figure 5: Characterization of CD80 expression and localization in a mouse model of inflammation-driven colon carcinogenesis. (A) Scheme for the experimental course of the colon carcinogenesis model. Mice were injected with AOM i.p. at a dose of $7.4 \mathrm{mg} / \mathrm{kg}$ body weight. After 4 weeks, mice were treated with $2.5 \%$ DSS in drinking water for 5 days, followed by 2 weeks of regular water. This cycle was repeated 2 more times. Animals were sacrificed at different time points $(0,7,28,56$ days) after the end of the last DSS cycle. (B) Flow cytometric analysis of CD80+ intestinal epithelial cells and quantification of (C) the inflammatory score, (D) LGD and (E) HGD extension during the time course of the inflammation-driven colon carcinogenesis model $(n=6-10$ mice per group). (F) Representative immunohistochemical staining for CD80 of the colon from AOM/DSS treated mice exhibiting CD80+ cells in the normal epithelium, LGD and HGD areas. Arrows indicate CD80 staining on the epithelial surface $(\uparrow)$, colonic crypts $(\leftarrow)$ and lamina propria $(\downarrow)$. The frequency of CD80 positive loci present in the colonic crypts, epithelial surface and lamina propria is shown. Data are presented as mean \pm S.E.M.. The Kruskal-Wallis test was used for comparisons.

differential expression in the healthy mucosa of UC patients with cancer as compared to the mucosa of UC patients without cancer [26]. Taken together, these observations suggest that, in the early stages of neoplastic transformation, the intestinal epithelium might show a distinct antigenic profile which, in association to CD80 and HLA-ABC, may be recognised and eliminated by the immune system.

An active role in the immune surveillance mechanisms of CD8+ lymphocytes expressing CD28, the CD80 receptor essential for T cell activation [27], is supported by their diminished number at the cancer stage in patients with UC. Koch et al. demonstrated a tumorspecific activation and cytotoxic activity of CD8+ tumour infiltrating lymphocytes (TIL) against tumor antigens in early CRC and several authors had already reported a down-regulation of CD8 activation and infiltration in advanced CRC [19, 28-30]. Moreover, among patients with $\mathrm{CRC}$, an apparent 'protective' function associated to high CD8+ lymphocyte infiltration was enhanced by nuclear expression of IL-23p19 within cancer cells [31]. Our data indicate that UC-related precancerous lesions are an even more effective stimulus to activate CD8+ lymphocytes. Moreover, the synchronous presence of CD80+ epithelial cells and activated CD8+ T-cells suggests that the immune surveillance mechanism in UC may be mediated by the direct interaction between epithelial cells and mucosal CD8+ T-cells. Indeed, Hodge et al. demonstrated that tumor cells transduced with CD80 gene by means of a retroviral vector became susceptible to $\mathrm{T}$ cell-mediated elimination [32]. 
A
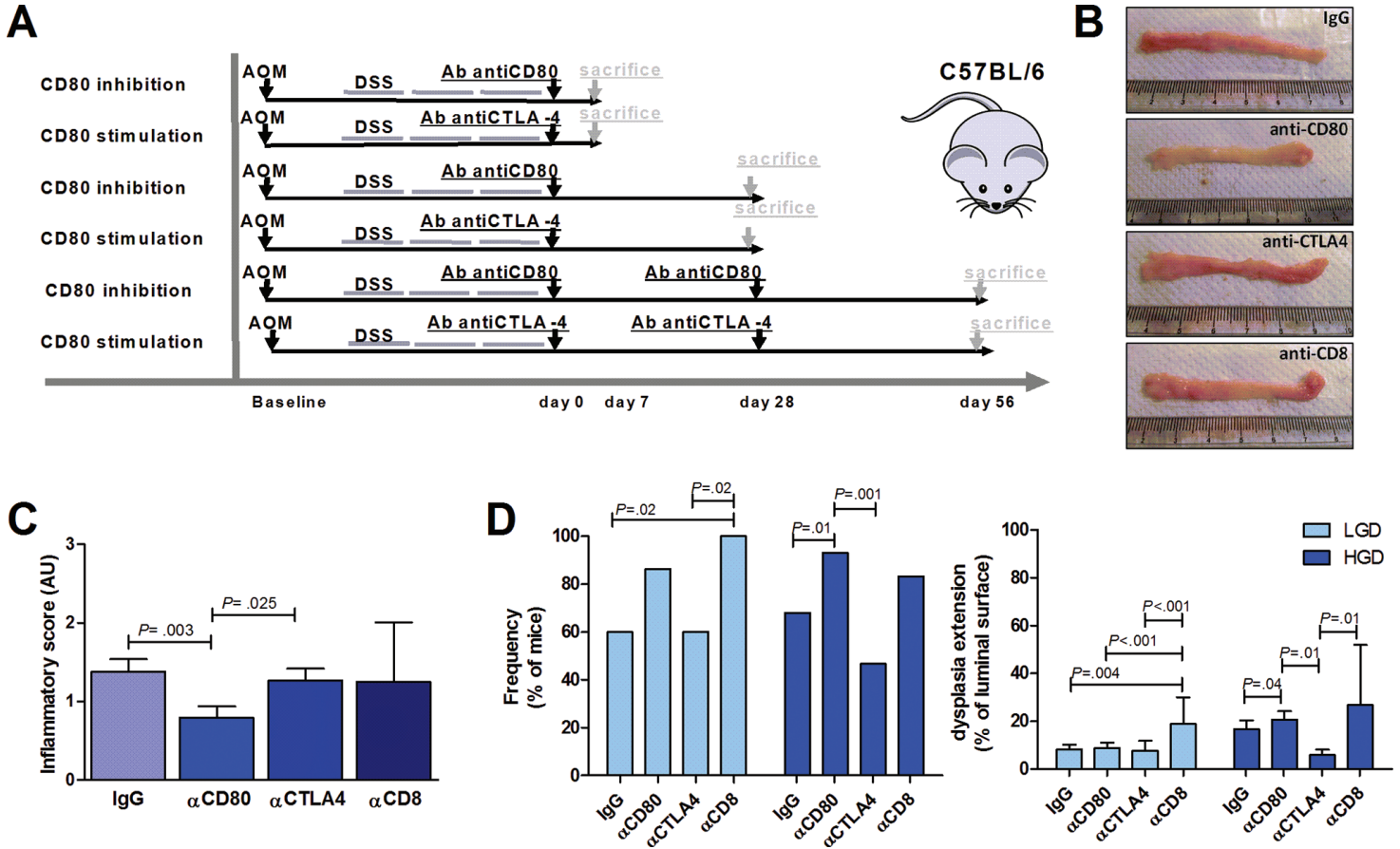

Figure 6: Effect of CD80 signaling modulation on the progression of an inflammation-driven colon carcinogenesis model. (A) Scheme for the experimental course of the colon carcinogenesis model for the administration of neutralizing antibodies (200 $\mu \mathrm{g} / \mathrm{mouse}$ ) against CD80, CTLA4 or CD8. (B) Representative images of colons from AOM/DSS-treated mice sacrificed at day 28 after neutralizing antibodies administration. (C) Cumulative inflammatory scores of AOM/DSS-treated mice subjected to the administration of control IgG, anti-CD80, anti-CTLA4 or anti-CD8 antibodies ( $n=6-25$ mice per group). (D) Cumulative frequency and extension of LGD and HGD in AOM/DSS-treated mice subjected to administration of IgG, anti-CD80, anti-CTLA4 or anti-CD8 antibodies $(n=6-25$ mice per group). The Kruskal-Wallis ANOVA followed by Mann-Whitney $U$ test were used for comparisons.

To show the ability of IEC to directly modulate lymphocyte activation through CD80 signaling, we co-cultured IEC from healthy colonic mucosa with syngeneic lymphocytes extracted from pericolonic lymph nodes of patients undergoing surgery for non neoplastic disease and for colonic adenocarcinoma. A limitation of this assay was that we used IEC from patients with Crohn's disease and diverticulitis in remission instead of those from UC patients. Indeed, we deliberately used them because non inflamed colonic tissue can be much easily retrieved from surgical specimen of non UC-patients. IEC isolated from patients with non neoplastic disease provoked an increase in the proportion of activated $\mathrm{CD} 8+\mathrm{CD} 38+$ T-cell that was significantly blocked by the addition of anti-CD80 antibody, whereas no significant changes were observed in terms of CD4 activation. By contrast, IEC isolated from neoplastic colon failed to activate CD8+ T-cells, supporting the view that APC function or CD8+ T-cell function (or both) are inhibited in presence of invasive cancer [33]. This is consistent with a previous study showing that stimulation with mucosal homogenate from intestinal segments containing dysplastic epithelium resulted in vigorous proliferation of $\mathrm{T}$ lymphocytes from mesenteric nodes draining the dysplastic-colonic region [33].
To begin to dissect the in vivo functional role of CD80 in colonic inflammatory carcinogenesis, we used AOM-DSS colitis as a mouse model of inflammatory colonic carcinogenesis $[34,35]$. The prevalence of CD80+ epithelial foci in correspondence of LGD or HGD, as opposed to the homogeneously distributed CD80+ lamina propria mononucleated cells in the presence of normal or dysplastic epithelium, further suggests that epithelial cells, in addition to professional APCs, are involved in the cross-talk with T lymphocytes in colon carcinogenesis. Interestingly, the number of CD80+ epithelial cells followed a trend similar to that of the inflammatory score over time, whereas an opposite trend between CD80 expression and HGD extension was observed, thus evidencing the possibility that either inflammation and tumorigenic stimuli contribute to the regulation of CD80 expression in this experimental model with distinct chronological patterns. Consistent with a pivotal role for CD80 in cancer immune surveillance, neutralization of CD80 caused a significant increase in HGD, whereas enhancing CD80 signaling with anti-CTLA4 protected mice from colon carcinogenesis progression. Together, these data confirm that CD80 signaling plays a prominent role in the immune surveillance mechanisms, as CD80 impairment enables the progression from LGD to HGD without 

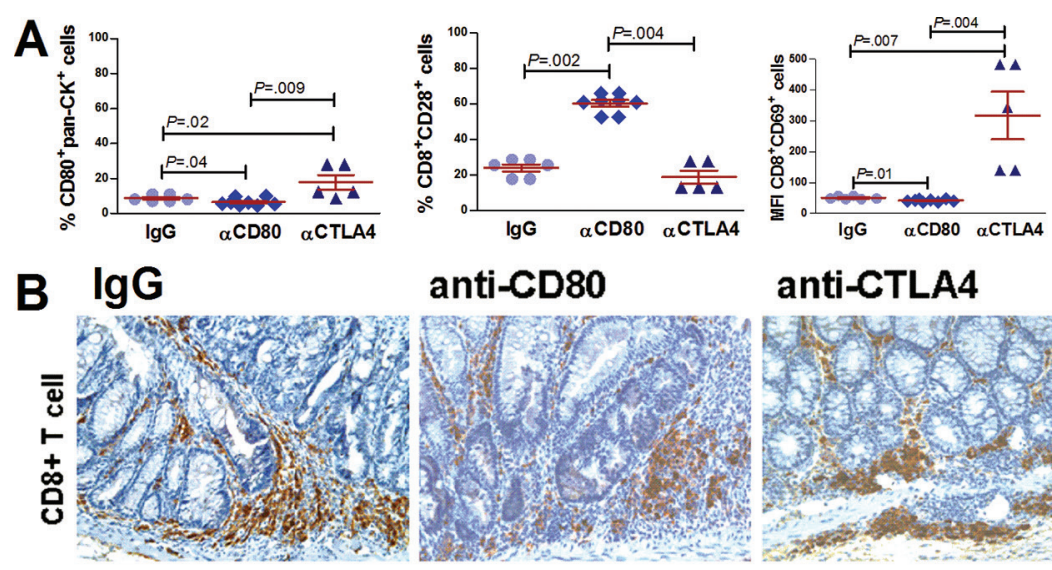

anti-CD80
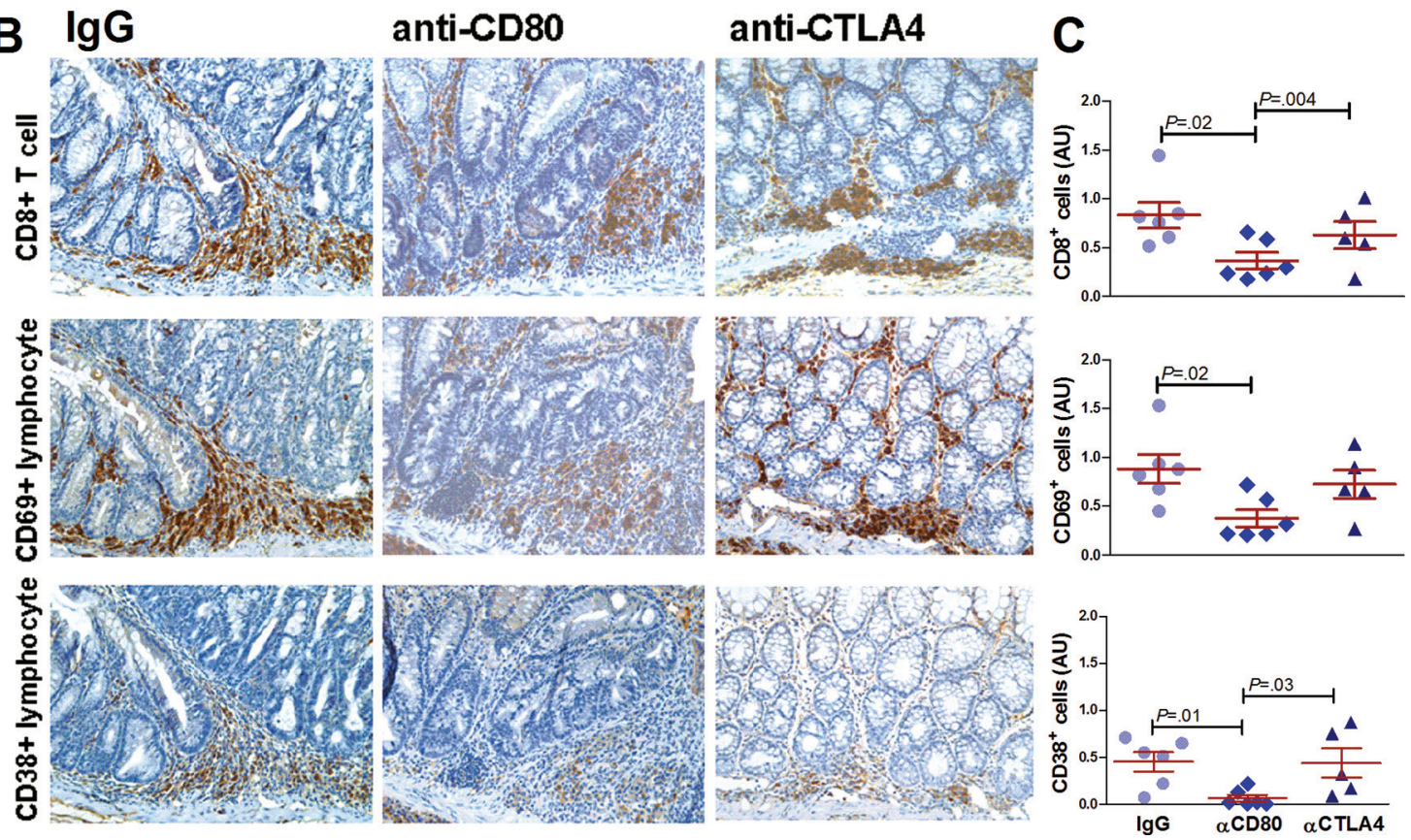

Figure 7: Effect of CD80 signaling modulation on CD8+ lymphocyte activation in a mouse model of inflammationdriven colon carcinogenesis. (A) Flow cytometric analysis of CD80+ epithelial cells (pan-ck+), CD8+CD28+ and CD8+CD69+ lymphocytes from the colons of AOM/DSS-treated mice subjected to i.p. administration of $200 \mu \mathrm{g}$ of control IgG, anti-CD80 or anti-CTLA4 antibodies. Animals were sacrificed on day 7 ( $n=5-6$ mice per group). Representative immunohistochemical staining (B) and quantification (C) of CD8, CD69 and CD38 cells of the colons of AOM/DSS-treated mice subjected to the administration of control IgG, anti-CD80 or anti-CTLA4 antibodies sacrificed at day 7 ( $n=5-6$ mice per group) (40x magnification). Data are presented as the mean \pm S.E.M. The Kruskal-Wallis ANOVA followed by Mann-Whitney $U$ test were used for comparisons.

affecting the appearance of LGD foci. In our opinion, in HGD, but not LGD, antigens that epithelial cells can present in association with CD80 are specifically expressed. However, CD80 signaling augmentation by means of anti-CTLA4 administration failed to completely eradicate the dysplasia. This might be explained by the AOM-DSS carcinogenesis model mechanism through random DNA damage that might involve CD80 gene regulation or the regulation of other genes involved in the CD80 signal cascade.

The analysis of the immunological events occurring early after the inflammatory insult in our model of CRC revealed that CD80-CD28 signaling pathway blockade results in inadequate lymphocytes activation that might induce antigen-specific tolerance and tumor progression [36]. By contrast, anti-CTLA4 treatment increased the proportion of CD80+ epithelial cell and the expression of CD69 on CD8+ T cells. Indeed, the presence of activated CD8+ lymphocytes in dysplastic areas suggests that these cells may be the main executors of the CD80 immune surveillance process in the colon. In line with this view,
CD8 neutralization caused a significant increase in HGD extension and frequency similarly to CD80 inhibition, proving that cytotoxic lymphocytes can control the progression of the early stages of inflammatory colonic carcinogenesis.

Taken together, our data support the hypothesis that in colonic inflammatory carcinogenesis the progression from dysplasia to invasive cancer is controlled not by a mere immunoediting process, such as that observed in sporadic invasive cancer [30], but by a truly effective immune surveillance mechanism mediated by CD80 expression on epithelial cells that can completely clear preneoplastic lesions in a large proportion of cases. The dysregulation of this immune checkpoint may lead to the progression from LGD to HGD and invasive cancer in UC [29]. Thus, blocking antibodies against CTLA4 such as ipilimumab, already approved for clinical use in melanoma patients to down regulate tumor-induced tolerance and immune suppression [37], might find a clinical application in colonic carcinogenesis [38]. To our knowledge, only one phase II trial with anti-CTLA4 
monoclonal antibody has been conducted on advanced CRC patients [39]. The lack of significant clinical activity reported in this trial may be explained by the fact that only one patient (out of 47) received more than one dose of antibody. Although a relatively poor-prognosis group of heavily pretreated patients was recruited, $43 \%$ of these patients were still alive at 6 months [39]. Moreover, in studies on advanced melanoma patients ipilimumab did not change median survival but, in the subgroup of patients who responded, it significantly prolonged survival over any expectation [37].

In conclusion, this study provide new insights about the mechanisms of immune surveillance in UCassociated colon carcinogenesis, thus having a potential impact in the early risk prediction and prevention of colon cancer. Future clinical studies will be required to evaluate the potential value of the use of immunotherapy in combination with other conventional treatment approaches to prevent, control, and/or eradicate established colon cancer.

\section{MATERIALS AND METHODS}

\section{Patients}

A prospective cohort of 153 patients who completed a colonoscopy or underwent colonic resection for either UC (with or without colonic dysplasia or colon cancer), colonic adenoma or CRC was enrolled. Furthermore, 47 healthy subjects who underwent colonoscopy for colonic cancer screening were recruited as controls. Mucosal samples were obtained from colonic biopsies of normal (sigmoid colon), neoplastic or preneoplastic mucosa (macroscopic lesion). Diagnosis was confirmed by clinical, radiological and histological parameters. The UC patients were receiving 5-ASA or steroids; none of the patients were treated with azathioprine, cyclosporine or anti-TNF $\alpha$ within the last six months. Moreover, patients treated with chemo- or radiotherapy in the previous six months were excluded. The study was performed according to the principles of the Declaration of Helsinki, all participants provided informed consent, and IRB approval (Veneto Institute of Oncology, Padova, Italy) was obtained. The characteristics of the patients and controls are outlined in Supplementary Table 1.

\section{Isolation of human intestinal epithelial cells (IEC) and mesenteric lymph node (MLN) lymphocytes}

Human IEC and MLN lymphocytes were isolated from normal colonic tissue and mesenteric lymph nodes, respectively, obtained from patients undergoing surgery for CRC, ileal Crohn's disease or diverticulitis. IEC were isolated and cultured as previously reported [40]. See Supplementary Methods for details.

\section{IEC:MLN lymphocytes co-culture assay}

Human MLN lymphocytes were suspended at $1 \times$ $10^{6}$ cells $/ \mathrm{ml}$ in DMEM with $10 \%$ FBS, $2.5 \%$ PenicillinStreptomycin-Fungizone and 1\% Gentamicin and cultured either alone or with syngeneic primary human IEC for 24 hours, in the presence or absence of neutralizing antibodies against CD80 (eBioscience). After the culture period, lymphocytes were harvested by vigorous pipetting and washed three times with PBS. Cells were then stained for CD4, CD8 and CD38 and analyzed by flow cytometry.

\section{Mouse colon cancer model}

Animal experiments were performed according to Italian Law 116/92 and European directive 2010/63/UE. Experimental protocols were reviewed and approved by the Institutional Animal Care and Use Committee ("Comitato Etico Scientifico per la Sperimentazione Animale") of the University of Padova, Padova, Italy. Mice were maintained under standard laboratory conditions with 12:12-h light-dark cycles and free access to regular rodent chow food and water at all stages of the experimental model. We obtained male 8-week-old C57B16/J mice from Harlan Laboratories S.r.l. Cohoused 10-wk-old mice were injected with azoxymethane (AOM, Sigma) i.p. at a dose of $7.4 \mathrm{mg} / \mathrm{kg}$ body weight (mean weight $27 \pm 1 \mathrm{gr}$ ). After 4 weeks, mice received $2.5 \%$ dextran sodium sulphate (DSS, Applichem) (M.W. 40,000 g/mol) in the drinking water for 5 days, followed by 2 weeks of regular water. This cycle was repeated two more times [34].

Mice were randomized into four groups and treated i.p. with $200 \mu \mathrm{g} /$ mouse of either control IgG, anti-CD80 $\mathrm{Ab}$ (clone 16-10A1, ATCC hybridoma no. HB-301), antiCTLA4 Ab (clone UC10-4F10-11, ATCC hybridoma no. HB-304) or anti CD8 (clone 2.43, ATCC hybridoma no. TIB-210) monthly, starting one week after the third DSS treatment [35, 41]. All mice were housed in the same animal room and sacrificed at different time points ( 1 week, 4 weeks and 8 weeks) after the first antibody injection. Colons were removed, flushed with PBS and a segment of the most distal colon above the anus was collected and processed for flow cytometric analysis. Remaining tissue was fixed as "Swiss rolls" in 10\% neutral-buffered formalin and paraffin embedded for histology.

\section{Histopathology}

Sections $(3 \mu \mathrm{m})$ from formalin-fixed and paraffinembedded human and mice specimens were stained with hematoxylin-eosin. Histological inflammation was quantified and classified by a pathologist (S.M.) unaware of the arm of the experiment using Floren's score [42] and the Vienna classification of gastrointestinal epithelial neoplasia [43]. The classification of epithelial neoplasia was supported by Ki67 staining (Supplementary Figure 2). 
Murine colons were analyzed for dysplasia at high magnification (40x). The extent of dysplasia was quantified as the percentage of involved bowel length. Inflammation was scored from 0 to 4 , with normal colonic mucosa $=$ 0 , shortening and loss of the basal one-third of the crypts with mild inflammation in the mucosa $=1$, loss of the basal two-thirds of the crypts with moderate inflammation in the mucosa $=2$, loss of the entire crypts but not the surface epithelium with severe inflammation in the mucosa $=3$, and loss of both entire crypts and surface epithelium with severe inflammation in the mucosa $=4$ [44].

\section{Immunohistochemistry}

Immunohistochemical analyses were performed using standard procedures, and the resulting sections were evaluated by a single pathologist in a blinded fashion. CD80 expression was graded on a semi-quantitative scale (negative, low, moderate or high). The frequency of CD80+ cells was assessed by counting the number of positive cells in a hundred cells in a representative field (LGD, HGD and normal epithelium). Ten random fields (40x) from each sample were examined. The expression of CD8, CD69 and CD38 was measured semi-quantitatively (none, mild, moderate and severe infiltration). The number of fields (40x) with moderate/ severe infiltration per intestinal surface was obtained and considered a parameter of the immune response to early epithelial mutations. The antibodies used are summarized in Supplementary Table 2.

\section{RNA extraction and $q R T-P C R$}

Total RNA from intestinal tissue was extracted using the SV Total RNA Isolation System (Promega) according to the manufacturer's instructions. Complementary DNA (cDNA) synthesis was performed using the Applied Biosystems cDNA Synthesis kit according to the manufacturer's directions. Specific mRNA transcripts were quantified with SYBR Green PCR Master Mix in an ABI PRISM 7000 Sequence Detection System (Applied Biosystems). The expression of the target molecule was normalized to the expression of the Actab housekeeping gene. The primers used are summarized in Supplementary material.

\section{Immunoassay}

Tissues were mechanically homogenized in $500 \mu \mathrm{l}$ of PBS (1:10 wt/vol ratio) containing a protease inhibitor cocktail (Roche Applied Science). Protein concentration was determined using the BCA Protein Assay kit (Pierce). Mucosal levels of IL-12, TNF- $\beta$, granzyme B (Bender MedSystems $\mathrm{GmbH}), \mathrm{IL}-17$ (R\&D Systems), T-bet (CUSABIO) and IL35 (Uscn Life Science Inc) were measured by immunometric assay according to manufacturer's instructions. Data are expressed in pg per $\mu \mathrm{g}$ of total proteins.

\section{Flow cytometry}

Human intestinal mucosa was stripped from the muscularis mucosa, cut into strips, and freed of mucus by a 30-min wash in HBSS containing $10 \mathrm{mM}$ DTT (Applichem). IEC were isolated by $30-\mathrm{min}$ incubation of the intestinal mucosa in HBSS containing $1 \mathrm{mM}$ EDTA (Sigma-Aldrich). For LPMC, stripped mucosa, freed of mucus and IEC, was digested with $1 \mathrm{mg} / \mathrm{ml}$ collagenase and DNase (Sigma Aldrich) for $30 \mathrm{~min}$ at $37^{\circ} \mathrm{C}$. The resulting crude cell suspensions were purified using a Ficoll-Hypaque Plus gradient (GE Healthcare), and the preparations were preferentially enriched for LPMC, washed, and collected. Mice colonic samples were freed of mucus by a $30-\mathrm{min}$ wash in HBSS containing $10 \mathrm{mM}$ DTT (Applichem). Then the mucosa was removed by the underlying muscle layer, and digested with $1 \mathrm{mg} / \mathrm{ml}$ collagenase and DNase (Sigma Aldrich) for $30 \mathrm{~min}$ at $37^{\circ} \mathrm{C}$. Preparations were then enriched preferentially for LPMC using a Ficoll-Hypaque Plus gradient (GE Healthcare).

For staining of freshly isolated cells, 105 cells were stained in $\mathrm{PBS} / 2 \%$ FBS with appropriate combinations of FITC- and PE-conjugated antibodies for $30 \mathrm{~min}$ on ice. Single-cell suspensions were subjected to flow cytometry to determine the proportion of epithelial cells (Cytokeratin 20, Cyt-20+) acting as antigen-presenting cells (expressing CD80, CD40, HLA ABC or HLA DR) and the proportion of activated CD8+ T cells (positive for CD28, CD38 or CD69). Flow cytometric analysis was performed using a FACSCalibur based on CellQuest software (Becton Dickinson). The antibodies used are summarized in Supplementary Table 2.

\section{Statistics}

Data are presented as the mean+/- SEM. The nonparametric Mann-Whitney's U-test for independent variables, the Wilcoxon test for paired matched variables or Kruskall-Wallis ANOVA for multiple variables were used for comparisons as appropriate. The Kendall rank correlation test was performed. Differences were considered significant at $P<0.05$.

\section{ACKNOWLEDGMENTS}

Funding This study was supported by the Current Research Funds from the Veneto Institute of Oncology IOV - IRCCS and by the Funds of Finalized Research 2010 (project MICCE1) from the Veneto Region; both were assigned to Carlo Castoro.

\section{Precis}

In this study we observed that human colonic epithelial cells can act as antigen presenting cells in 
UC-related carcinogenesis leading to CD8 $\mathrm{T}$ cell activation and we demonstrated that modulation of CD80CD28 signaling in a murine model of colitis-associated carcinogenesis can alter the progression from LGD to HGD.

\section{Competing interests}

None

\section{Conflict of interest statement}

The authors disclose no potential conflicts of interest.

\section{REFERENCES}

1. Munkholm P. The incidence and prevalence of colorectal cancer in inflammatory bowel disease. Aliment Pharmacol Ther. 2003; 18:1-5.

2. Eaden JA, Abrams KR, Mayberry JF. The risk of colorectal cancer in ulcerative colitis: a meta-analysis. Gut. 2001; 48:526-535.

3. Lutgens MW, Vleggaar FP, Schipper ME, Stokkers PC, van der Woude CJ, Hommes DW, de Jong DJ, Dijkstra G, van Bodegraven AA, Oldenburg B, Samsom M. High frequency of early colorectal cancer in inflammatory bowel disease. Gut. 2008 Sep; 57:1246-51.

4. Itzkowitz S. Colon carcinogenesis in inflammatory bowel disease. J Clin Gastroenterol. 2003; 36:S70-4.

5. Serafino A, Moroni N, Zonfrillo M, Andreola F, Mercuri L, Nicotera G, Nunziata J, Ricci R, Antinori A, Rasi G, Pierimarchi P. WNT-pathway components as predictive markers useful for diagnosis, prevention and therapy in inflammatory bowel disease and sporadic colorectal cancer. Oncotarget. 2014 Feb 28; 5:978-92.

6. Van Assche G, Dignass A, Bokemeyer B, Danese S, Gionchetti P, Moser G, Beaugerie L, Gomollón F, Häuser W, Herrlinger K, Oldenburg B, Panes J, Portela F, et al; European Crohn's and Colitis Organisation. Second European evidence-based consensus on the diagnosis and management of ulcerative colitis part 3: special situations. J Crohns Colitis. 2013 Feb; 7:1-33.

7. Lim CH, Dixon MF, Vail A, Forman D, Lynch DA, Axon AT. Ten year follow up of ulcerative colitis patients with and without low grade dysplasia. Gut. 2003 Aug; 52:1127-32.

8. Lynch DA, Lobo AJ, Sobala GM, Dixon MF, Axon AT. Failure of colonoscopic surveillance in ulcerative colitis. Gut. 1993 Aug; 34:1075-80.

9. Kiran RP, Ahmed Ali U, Nisar PJ, Khoury W, Gu J, Shen B, Remzi FH, Hammel JP, Lavery IC, Fazio VW, Goldblum JR. Risk and location of cancer in patients with preoperative colitis-associated dysplasia undergoing proctocolectomy. Ann Surg. 2014 Feb; 259:302-9.

10. Zisman TL, Bronner MP, Rulyak S, Kowdley KV, Saunders M, Lee SD, Ko C, Kimmey MB, Stevens A, Maurer J, Brentnall TA. Prospective study of the progression of low-grade dysplasia in ulcerative colitis using current cancer surveillance guidelines. Inflamm Bowel Dis. 2012 Dec; 18:2240-6.

11. Dunn GP, Koebel CM, Schreiber R.D. Interferons, immunity and cancer immunoediting. Nat Rev Immunol. 2006; 6:836-848.

12. Chen L, Ashe S, Brady WA, Hellström I, Hellström KE, Ledbetter JA, McGowan P, Linsley PS. Costimulation of antitumor immunity by the B7 counterreceptor for the T lymphocyte molecules CD28 and CTLA-4. Cell. 1992 Dec 24; 71:1093-102.

13. Janeway CA, Bottomly K. Signals and signs for lymphocyte responses. Cell. 1994; 76:275-285.

14. Schwartz RH. A cell culture model for T lymphocyte clonal anergy. Science. 1990; 248:1349-1356.

15. Leach DR, Krummel MF, Allison JP. Enhancement of antitumor immunity by CTLA-4 blockade. Science. 1996; 271:1734-1736.

16. Antonia SJ, Muñoz-Antonia T, Soldevila G, Miller J, Flavell RA. B7-1 expression by a non-antigen presenting cell-derived tumor. Cancer Res. 1995 Jun 1; 55:2253-6.

17. Scarpa M, Cardin R, Bortolami M, Kotsafti A, Scarpa MC, Pozza A, Maran G, Picciocchi M, Ruffolo C, D'Incà R, Sturniolo GC, Castagliuolo I, Castoro C, et al. Mucosal immune environment in colonic carcinogenesis: CD80 expression is associated to oxidative DNA damage and TLR4-NFkB signalling. Eur J Cancer. 2013; Jan 49:254-63.

18. Scarpa M, Behboo R, Angriman I, Cecchetto A, D'Incà R, Termini B, Barollo M, Ruffolo C, Polese L, Sturniolo GC, D'Amico DF. Expression of costimulatory molecule CD80 in colonic dysplasia in ulcerative colitis: an immunosurveillance mechanism against colorectal cancer?. Int J Colorectal Dis. 2006 Dec; 21:776-83.

19. Koch M, Beckhove P, Op den Winkel J, Autenrieth D, Wagner P, Nummer D, Specht S, Antolovic D, Galindo L, Schmitz-Winnenthal FH, Schirrmacher V, Büchler MW, Weitz J. Tumor infiltrating $\mathrm{T}$ lymphocytes in colorectal cancer: Tumor-selective activation and cytotoxic activity in situ. Ann Surg. 2006 Dec; 244:986-92; discussion 992-3.

20. Pagès F, Berger A, Camus M, Sanchez-Cabo F, Costes A, Molidor R, Mlecnik B, Kirilovsky A, Nilsson M, Damotte D, Meatchi T, Bruneval P, Cugnenc PH, et al. Effector memory T cells, early metastasis, and survival in colorectal cancer. $\mathrm{N}$ Engl J Med. 2005 Dec 22; 353:2654-66.

21. Burnet FM. The concept of immunological surveillance. Prog Exp Tumor Res. 1970; 13:1-27.

22. Dunn GP, Bruce AT, Ikeda H, Old LJ, Schreiber RD. Cancer immunoediting: from immunosurveillance to tumor escape. Nat Immunol. 2002 Nov; 3:991-8.

23. Koebel CM, Vermi W, Swann JB, Zerafa N, Rodig SJ, Old LJ, Smyth MJ, Schreiber RD. Adaptive immunity maintains occult cancer in an equilibrium state. Nature. 2007 Dec 6; 450:903-7. 
24. Pages F, Galon J, Fridman WH. The essential role of the in situ immune reaction in human colorectal cancer. J Leukocyte Biol. 2008; 84:981-987.

25. Scarpa M, Bortolami M, Cecchetto A, Faggian D, Kotsafti A, Ruffolo C, Navaglia F, Pozza A, D'Incà R, Plebani M, Sturniolo GC, Angriman I. Mucosal immune environment in colonic carcinogenesis: CD80 up-regulation in colonic dysplasia in ulcerative colitis. Eur J Cancer. 2011 Mar; 47:611-9.

26. Watanabe $\mathrm{T}$, Kobunai $\mathrm{T}$, Yamamoto $\mathrm{Y}$, Ikeuchi $\mathrm{H}$, Matsuda K, Ishihara S, Nozawa K, Iinuma H, Kanazawa T, Tanaka T, Yokoyama T, Konishi T, Eshima K, et al. Predicting ulcerative colitis-associated colorectal cancer using reverse-transcription polymerase chain reaction analysis. Clin Colorectal Cancer. 2011 Jun; 10:134-41.

27. Evans EJ, Esnouf RM, Manso-Sancho R, Gilbert RJ, James JR, Yu C, Fennelly JA, Vowles C, Hanke T, Walse B, Hünig T, Sørensen P, Stuart DI, et al. Crystal structure of a soluble CD28-Fab complex. Nat Immunol. 2005 Mar; 6:271-9.

28. Galon J, Costes A, Sanchez-Cabo F, Kirilovsky A, Mlecnik B, Lagorce-Pagès C, Tosolini M, Camus M, Berger A, Wind P, Zinzindohoué F, Bruneval P, Cugnenc $\mathrm{PH}$, et al. Type, density, and location of immune cells within human colorectal tumors predict clinical outcome. Science. 2006 Sep 29; 313:1960-4.

29. Pardoll DM. The blockade of immune checkpoint in cancer immunotherapy. Nat Rev Cancer. 2012; 12:252-264.

30. Hodge JW, Abrams S, Schiom J, Kantor JA. Induction of antitumor immunity by recombinant vaccinia viruses expressing B7-1 or B7-2 costimulatory molecules. Cancer Res. 1994; 54:5552-5555.

31. Helbling M, Lukesch A, Haimovici A, Karamitopoulou E, Berger MD, Hädrich M, Mallaev M, Schnüriger B, Koelzer VH, Dawson H, Borner M, Langer R, Rosenberg R, et al. Investigation of IL-23 (p19, p40) and IL-23R identifies nuclear expression of IL-23 p19 as a favorable prognostic factor in colorectal cancer: a retrospective multicenter study of 67 patients. Oncotarget. 2014 Jul 15; 5:4671-82.

32. Malavasi F, Deaglio S, Funaro A, Ferrero E, Horenstein AL, Ortolan E, Vaisitti T, Aydin S. Evolution and function of the ADP ribosyl cyclase/CD38 gene family in physiology and pathology. Physiol Rev. 2008 Jul; 88:841-86.

33. Karlsson $M$, Lindberg $K$, Karlén P, Ost A, Thörn M, Winqvist O, Eberhardson M. Evidence for immunosurveillance in intestinal premalignant lesions. Scand J Immunol. 2010 May; 71:362-8.

34. De Robertis M, Massi E, Poeta ML, Carotti S, Morini S, Cecchetelli L, Signori E, Fazio VM. The AOM/DSS murine model for the study of colon carcinogenesis: From pathways to diagnosis and therapy studies. J Carcinog. 2011 Mar 24; 10:9.

35. Eri R, Kodumudi KN, Summerlin DJ, Srinivasan M. Suppression of colon inflammation by CD80 blockade: evaluation in two murine models of inflammatory bowel disease. Inflamm Bowel Dis. 2008 Apr; 14:458-70.

36. Rudd CE, Taylor A, Scheider H. CD28 and CTLA4 coreceptor expression and signal transduction. Immunol Rev. 2009; 229:12-26.

37. McDermott D, Haanen J, Chen TT, Lorigan P. Efficacy and safety of ipilimumab in metastatic melanoma patients surviving more than 2 years following treatment in a phase III trial (MDX010-20). Ann Oncol. 2013 Oct; 24:2694-8.

38. Zang X, Allison JP. The B7 family and cancer therapy: costimulation and coinhibition. Clin Cancer Res. 2007; 13:5271-9.

39. Chung KY, Gore I, Fong L, Venook A, Beck SB, Dorazio P, Criscitiello PJ, Healey DI, Huang B, GomezNavarro J, Saltz LB. Phase II study of the anti-cytotoxic T-lymphocyte-associated antigen 4 monoclonal antibody, tremelimumab, in patients with refractory metastatic colorectal cancer. J Clin Oncol. 2010 Jul 20; 28:3485-90.

40. Grossmann J, Walther $\mathrm{K}$, Artinger $\mathrm{M}$, Kiessling $\mathrm{S}$, Steinkamp M, Schmautz WK, Stadler F, Bataille F, Schultz M, Schölmerich J, Rogler G. Progress on isolation and short-term ex-vivo culture of highly purified nonapoptotic human intestinal epithelial cells (IEC). Eur J Cell Biol. 2003 May; 82:262-70.

41. Srinivasan M, Summerlin DJ. Modulation of the colonic epithelial cell responses and amelioration of inflammation by CD80 blockade in TNBS colitis. Clin Immunol. 2009; 133:411-421.

42. Florén $\mathrm{CH}$, Benoni C, Willén R. Histologic and colonoscopic assessment of disease extension in ulcerative colitis. Scand J Gastroenterol. 1987; 22:459-462.

43. Schlemper RJ, Riddell RH, Kato Y, Borchard F, Cooper HS, Dawsey SM, Dixon MF, Fenoglio-Preiser CM, Fléjou JF, Geboes K, Hattori T, Hirota T, et al. The Vienna classification of gastrointestinal epithelial neoplasia. Gut. 2000 Aug; 47:251-5.

44. Zisman TL, Bronner MP, Rulyak S, Kowdley KV, Saunders M, Lee SD, Ko C, Kimmey MB, Stevens A, Maurer J, Brentnall TA. Prospective study of the progression of low-grade dysplasia in ulcerative colitis using current cancer surveillance guidelines. Inflamm Bowel Dis. 2012 Dec; 18:2240-6. 